\title{
Stability of an Electrically Conducting Fluid Flow between Coaxial Cylinders under Magnetic Field
}

\author{
H. Benhacine ${ }^{1,3}$, B. Mahfoud ${ }^{2 \dagger}$ and M. Salmi ${ }^{3,4}$ \\ ${ }^{I}$ Department of Mechanical Engineering, University of Mohamed Boudiaf, M'sila,28000, Algeria \\ ${ }^{2}$ Materials and Sustainable Development Laboratory (LMDD), University of AMO-BOUIRA, 10000, Algeria \\ ${ }^{3}$ Laboratory of Physics and Chemistry of Materials, University of M'sila, Algeria. \\ ${ }^{4}$ Department of Physics, University of M'sila, B.P. 1713, M'sila, 28000, Algeria \\ $\dagger$ Corresponding Author Email: b.mahfoud@univ-bouira.dz
}

(Received May 27, 2021; accepted October 4, 2021)

\begin{abstract}
This research aims to investigate the vortex breakdown zone, the stability margin, and the fluid layers of the rotating flow between two vertical coaxial cylinders under the effect of thermal gradient and an axial magnetic field. The governing Navier-Stokes, temperature, and potential equations are solved using the finitevolume method. Three combinations of aspect ratios $(\gamma)$ and Reynolds numbers $(R e)$ are compared. The pumping action sets up a secondary circulation along the meridional plane of the annular gap. For certain combinations, the vortex breakdown bubble occurred near the inner wall. Bifurcation in form of multiple fluid layers becomes apparent when the temperature difference exceeds a critical value. These fluid layers play the role of thermal insulation and limit the heat transfer between the hot top and cold bottom of the coaxial cylinders. Both the vortex breakdown and fluid layers could be suppressed by the magnetic field; the increasing of Hartmann number $(\mathrm{Ha})$ would reduce the number of fluid layers. Diagrams represent the effect of increasing Richardson number $(R i)$ on fluid layers are established. Then stability diagrams corresponding to the transition from the multiple fluid layers zone to the one fluid layer zone for increasing Prandtl number $(P r)$ are obtained.
\end{abstract}

Keywords: Coaxial cylinders; Fluid layers; Heat transfer; Magnetic field; Vortex breakdown.

\section{NOMENCLATURE}

$B$

E

$\boldsymbol{F}_{L}$

$H$

$\mathrm{Ha}$

$\boldsymbol{J}$

$\overline{N u}$

$\overline{N u}$

$P$

Pr

$\operatorname{Re}$

$R e_{\mathrm{m}}$
$R i$

$R i$
$R$ magnitude of the external magnetic field, Tesla electric charges

Lorentz force

height of the cylinder, $\mathrm{m}$

Hartmann number

dimensionless current density

Nusselt number

average Nusselt number

dimensionless pressure

Prandtl number

Reynolds number

magnetic Reynolds number

Richardson number

annular gap, $\mathrm{m}$
$R_{\text {in }}$

$R_{o}$

$V$

$\alpha$

$\beta$

$\Theta$

$\gamma$

$v$

$\mu 0$

$\rho$

$\sigma$

$\Phi$

$\Omega$

$\Psi$ radius of the inner cylinder, $m$ radius of the outer cylinder, $m$ velocity vector thermal diffusivity of the fluid, $\mathrm{m}^{2} / \mathrm{s}$ thermal expansion coefficient, $1 / \mathrm{K}$ dimensionless temperature aspect ratio $\left(H / R_{o}\right)$ kinematic viscosity of the fluid, $\mathrm{m}^{2} / \mathrm{s}$ magnetic permeability, $\mathrm{H} / \mathrm{m}$ density of the fluid, $\mathrm{kg} / \mathrm{m}^{3}$ electric conductivity, $\Omega / m$ dimensionless electric potential angular velocity, $\mathrm{rad} / \mathrm{s}$ non-dimensional stream function dimensionless time

\section{INTRODUCTION}

Nuclear fusion reactors remain a good applicant for future power renewable sources. A liquid metal at a high temperature like the lead-lithium alloy $(\mathrm{PbLi})$

is used by magnetic confinement fusion (MCF) blankets, as a coolant/tritium breeder. The advancement of research in the domain of the LMMCF blankets requires the existence of knowledge that develops parallel to understanding the high- 
temperature liquid metals flows under the control of the plasma-confining magnetic field. Blankets with liquid walls should be of an elongated type. The coaxial configuration can be used by magnetic confinement fusion (MCF) blankets. This geometry has become interesting for experimental electrically conducting fluid studies with rotating motion. This geometry is particularly interesting to the researchers that adjust with precision the Reynolds number conducted on small annular gaps. Moreover, the flow pattern and the vortex breakdown can be observed by flow visualization techniques over a glass sidewall of the outer cylinder. The first visualization experiment exposed that this vortex undergoes breakdown which is presented by Vogel (1968) and draws also a curve showing the limits within each occurred vortex breakdown. Next, Escudier (1984) elaborates an experimental investigation using the technique of Laser-induced fluorescence to describe the internal flow structure. It is worth mentioning that several studies exist in the literature focusing on the examination of various methods used for controlling the vortex breakdown, such as the application of a temperature difference between two walls in a precise direction (Ismadi et al. 2011; Herrada and Shtern 2003). Crystal growth processing is one of many practical applications, which combines the rotating flow with heat transfer. The thermal gradient direction may be the cause of the increase or decrease of a layering flow (Omi and Iwatsu 2005; Mahfoud and Bessaïh 2012a) and a reduction in the Nusselt number (Turan et al. 2018; Quaresma et al. 2020). One of the implemented methods by researchers to control or suppress the breakdown is the use of magnetohydrodynamics. The literature review on convection problems confirmed that a magnetic field is used to stabilize the perturbation in the fluid motion and control the velocity field (Mahfoud and Bessaïh 2012b; 2016; Mahfoud et al.2016, 2019, 2020). After that, Bendjaghlouli et al. (2019a,b) have discussed the influence of the geometry and the thermal gradient to generated small vortices with and without an applied magnetic field. Other parameters such as the annular gaps effect and the electrical conductivity walls' effect on the structure of the flow have been studied by Kharicha et al. (2005). Some researchers have followed the position of the center and length of a vortex under a magnetic field, as revealed in the studies of (Yu et al. 2013; Dash and Singh 2019). Laouari et al. (2021) have recently studied the central position of the vortex and the limits of appearance and disappearance of the vortex breakdown in the different conductivity regimes. Mahfoud (2021a,b) confirmed that the vortex breakdown can be suppressed beyond the magnitude of the magnetic field to exceed a critical value.

In this paper, various numerical simulations are used to clarify the effects of the aspect ratio and rotation rate on vortex breakdown (apparition, and suppression). The second objective is to clarify the temperature gradient role on the appearance of the bifurcation in form of fluid layers between two coaxial vertical cylinders in which three viscous conducting fluids are compared. Finally, the aim is to specify the critical Hartmann numbers, $H a_{\mathrm{cr}}$ corresponding to removing the fluid layers. Therefore, we present the stability limits correspond to the domain where the layering does not occur. The flow is generated by the combined forces of buoyancy and the rotation of the bottom disk and is studied in the range of $0 \leq R i \leq 2$, and for three combinations (case A: $R e=1500, \gamma=1.5$ ); (case B: $R e=1855, \gamma=2.0$ ) and (case C: $R e=2400, \gamma=2.5$ and for only one annular gap $(R=0.9)$.

\section{FLOW CONFIGURATION AND MODEL}

A viscous conducting fluid rotates in the annular gap $(R)$ between two coaxial vertical cylinders with height $(H)$. The combined stabilizing action of the external magnetic field and thermal gradient are imposed in the vertical direction which is schematically plotted in Fig. 1. One annular gap $(R$ $=0.9)$ and three aspect ratio, $\gamma\left(H / R_{o}\right)=1.5,2.0$ and 2.5 are examined. The annular gap is defined as $(R$ $\left.=R_{o}-R_{\text {in }}\right)$, where $R_{\text {in }}, R_{o}$ are the radius of the internal and outer cylinders, respectively and $R_{o}=1.0$ in all cases. A temperature difference $(\Delta \mathrm{T})$ is axially imposed (the top disk is hotter than the bottom). The fluid and solid walls system are subjected to an external axial magnetic field, $\boldsymbol{B}$ $\left(B_{0} \mathbf{e}_{z}\right), \mathbf{e}_{z}$ is the unit vector in the z-direction. The lower disk rotates about the $\mathrm{z}$-axis at an angular velocity $\Omega$, which is supposed constant, while the upper disk is fixed. The magnetic Reynolds number $R e_{m}=\mu_{0} \sigma \Omega R_{o}^{2} \ll 1$, that measure the ratio of induction to magnetic diffusion. In this problem, when the fluid is characterized by a small Prandtl number, we can neglect the magnetic field induction by comparing it with the $B_{0}$. Also, the only effective force that remains is the electromagnetic force of Lorentz.

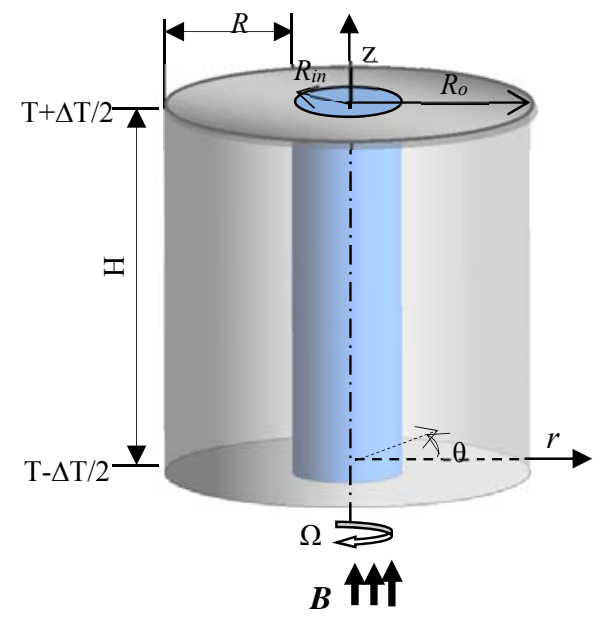

Fig. 1. Flow geometry.

For simplification of the problem, some assumptions are given: (i) three electrically conducting fluids considered here are incompressible and Newtonian;(ii) properties of 
fluids are constants and appraised at the reference temperature; except the density, which is treated according to Boussinesq's approximation, (iii) Joule heating, and viscous dissipation terms are neglected. Similarly, (iv) the container walls are electrically insulated, and (v) radiative heat transfer is also ignored in this calculation.

By scaling length with the the radius $R_{o}$ of the outer cylinder, time with $1 / \Omega$, velocities with $\Omega R_{o}$, pressure with $\rho\left(\Omega R_{o}\right)^{2}$, electric potential with $B_{0} \Omega R_{o}{ }^{2}$, and by introducing the dimensionless temperature as $\Theta=\left(\mathrm{T}-T_{0}\right) / \Delta \mathrm{T}$, the equations can be written in their dimensionless form as :

$\nabla \cdot V=0$

$\frac{\partial \boldsymbol{V}}{\partial \tau}+(\boldsymbol{V} . \nabla) \boldsymbol{V}=-\nabla P+\frac{1}{R e} \nabla^{2} \boldsymbol{V}+R i \Theta \boldsymbol{e}_{z}+$

$\frac{H a^{2}}{R e}(\boldsymbol{V} X \boldsymbol{B}) \times \boldsymbol{B}$

$\frac{\partial \Theta}{\partial \tau}+(\boldsymbol{V} . \nabla) \Theta=\frac{1}{R e \cdot P r} \nabla^{2} \Theta$

The induced electric current is given by the interaction of convective flow with the magnetic field, $\boldsymbol{J}=\sigma(\boldsymbol{E}+\boldsymbol{V} \times \boldsymbol{B})$ hence, the electric charges become $\boldsymbol{E}=-\nabla \Phi$. Where vector velocity $\mathbf{V}\left(\mathrm{v}_{r}\right.$, $\left.\mathrm{v}_{\theta}, \mathrm{v}_{z}\right)$, have the components in the radial $\left(\mathrm{v}_{r}\right)$, azimuthal $\left(\mathrm{v}_{\theta}\right)$ and axial $\left(\mathrm{v}_{z}\right)$ direction, respectively. The conservation of the induced electric current $\nabla \boldsymbol{J}=0$ gives the electric potential.

$\nabla^{2} \Phi=\nabla \cdot(\mathbf{V x} \boldsymbol{B})$

$P, \Theta$, and $\Phi$ are the dimensionless pressure, temperature, and electric potential, respectively. Non-dimensional parameters are Reynolds number $\left(R e=\Omega R_{0}{ }^{2} / \mathrm{v}\right)$; the Hartmann number ( $\left.H a=B_{0} R_{0} \sqrt{\sigma / \rho v}\right)$; the Richardson number $\left(R i=\beta g \Delta T / \Omega^{2} R_{0}\right)$. Two other non-dimensional parameters control the comportment of the flow: the aspect ratio $\left(\gamma=H / R_{o}\right)$ and the Prandtl number $(P r=v / \alpha)$. The symbols $\rho, v, \beta, \alpha$, and $\sigma$ denote, respectively, the density, the kinematic viscosity, the thermal expansion, the thermal diffusivity, and electric conductivity. The Lorentz force is given by $\boldsymbol{F}_{\boldsymbol{L}}=\boldsymbol{J} X \boldsymbol{B}$, which are in the $r, z$, and $\theta$ directions, respectively:

$\left\{\begin{array}{c}F_{L r}=-1 / r . \partial \Phi / \partial \theta-\mathrm{v}_{r} \\ F_{L z}=0 \\ F_{L \theta}=\partial \Phi / \partial r-\mathrm{v}_{\theta}\end{array}\right.$

With the current density $J$ is:

$\left\{\begin{array}{c}J_{r}=-\partial \Phi / \partial r+\mathrm{v}_{\theta} \\ J_{z}=-\partial \Phi / \partial z \\ J_{\theta}=-1 / r . \partial \Phi / \partial \theta-\mathrm{v}_{r}\end{array}\right.$

All simulations were performed starting from $\tau=0$. The bottom disk starts its rotation with angular velocity $\Omega$, while the top and side walls are stationary, respectively. The velocity field must satisfy the non-slip condition at the walls. All walls are electrically insulated $(\partial \Phi / \partial n=0)$.
The boundary conditions are at a rotating cold bottom disk.

$\mathrm{V}_{\theta}=r ; \quad \Theta=-\frac{1}{2}$

At a stationary hot top disk.

$\mathrm{v}_{\theta}=0$, and $\Theta=\frac{1}{2}$

Owing to the geometrical symmetry, the periodicity conditions are

$V(r, \theta, z)=V(r, \theta+2 \pi, z)$.

The velocity field is plotted in function of the nondimensional stream function $\psi$, which is :

$\mathrm{v}_{\mathrm{r}}=1 / r(\partial \Psi / \partial z), \mathrm{v}_{\mathrm{z}}=-1 / r(\partial \Psi / \partial r)$

The heat transfer and convection mode are concluded from the average Nusselt number which is calculated at the bottom disk as:

$\overline{N u}=\left(\frac{1}{\pi}\right) \int_{0}^{1} \int_{0}^{2 \pi} N u(r, \theta) r d \theta d r$

Where,

$N u(r, \theta)=\left.\left(\frac{\partial \Theta}{\partial z}\right)\right|_{z=0 \text { or } z=H}$

\section{NUMERICAL TECHNIQUE AND GRID EMPLOYED}

The governing equations and boundary conditions of the system mentioned above are discretized by the finite volume method and solved by the tridiagonal matrix algorithm (TDMA). SIMPLER scheme Patankar (1980) was selected to solve the coupling between velocity and pressure. The central-difference approximation scheme is used for the diffusion and convective terms.

In this simulation, three staggered nonuniform meshes were applied to get better convergence and are listed in Table.1. Taking into account that the thickness of the Hartmann layer $\left(\sim \mathrm{Ha}^{-1}\right)$, and the aspect $\operatorname{ratios}(\gamma)$, a careful choice of the grid is needed to ensure good accuracy. Moreover, the validity of the grid selections is confirmed via grid independence tests for three cases (A, B, C), using the average Nusselt number for comparison (see Table 2).

Table 1 Grid sizes used in the numerical

\begin{tabular}{|l|c|c|c|}
\hline $\begin{array}{l}\text { Aspect } \\
\text { ratios }\end{array}$ & $\begin{array}{l}\text { Case A } \\
\mathrm{Re}=1500, \\
\gamma=1.5\end{array}$ & $\begin{array}{l}\text { Case B } \\
\mathrm{Re}=1855, \\
\gamma=2.0\end{array}$ & $\begin{array}{c}\text { Case C } \\
\mathrm{Re}=2400, \\
\gamma=2.5\end{array}$ \\
\hline $\begin{array}{l}\text { Grid } \\
(r, \theta, z)\end{array}$ & $85 \times 85 \times 130$ & $85 \times 85 \times 170$ & $85 \times 85 \times 190$ \\
\hline
\end{tabular}

Table 2 Grid independence test for the case $(\mathrm{Pr}=$ $0.032, \mathrm{Ri}=0.1$, and $\mathrm{Ha}=5$ ).

\begin{tabular}{|c|c|c|c|}
\hline & \multicolumn{3}{|c|}{ Grid $(r, \theta, z)$} \\
\hline Case A & $80 \times 80 \times 120$ & $85 \times 85 \times 130$ & $90 \times 90 \times 140$ \\
\hline$\overline{N u}$ & 0.691 & 0.695 & 0.695 \\
\hline Case B & $80 \times 80 \times 160$ & $85 \times 85 \times 170$ & $90 \times 90 \times 180$ \\
\hline$\overline{N u}$ & 0.519 & 0.522 & 0.522 \\
\hline Case C & $80 \times 80 \times 180$ & $85 \times 85 \times 190$ & $90 \times 90 \times 200$ \\
\hline$\overline{N u}$ & 0.420 & 0.423 & 0.423 \\
\hline
\end{tabular}




\section{Results AND Discussion}

First, the used numerical model has been validated by comparing the present numerical results with the numerical results of Mahfoud et al. (2020) and Kakarantzas et al. (2017) who investigated the flow and heat transfer of liquid metal, which rotates in the annular of concentric cylinders under a magnetic field effect. Figure 2 shows the reproduction of the radial distribution of axial velocity in the middle of the domain ( $\mathrm{z}=1$ plane), for $H a=100$ and the aspect ratio $H / R_{0}=2.0$ when the annular gap is $R=0.4$ and the Rayleigh number $R a=10^{5}$. Although, there is a good agreement between the compared numerical results.

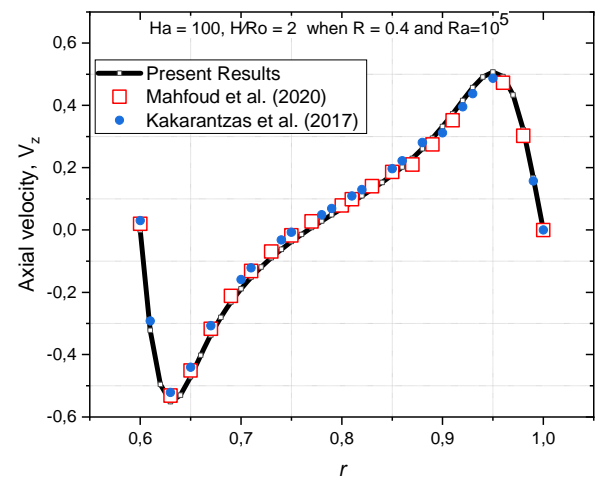

Fig. 2. Comparison with Mahfoud et al. (2020) and Kakarantzas et al. (2017).

The computations were performed for one annular gap $(R=0.9)$, and for three combinations of aspect ratios and Reynolds numbers (case $\mathrm{A}: R e=1500$, $\gamma=1.5$ ); (case B: $R e=1855, \gamma=2.0$ ) and (case C: $R e=2400, \gamma=2.5$ ). Three electrically conducting fluids are compared, the first one is characterized by Prandtl number, $P r=0.015$ corresponding to the molten Aluminum, where the Aluminum casting process still playing an important role in the modern industry. The second is the saturated liquid Mercury at $293 \mathrm{~K}$, corresponding to $P r=0.025$. Third, $\operatorname{Pr}=0.032$, corresponding to the PbLi 17 alloy, which is in the most appropriate position to ensure the fusion blankets. The Richardson number analyzed here covers the range of $0 \leq R i \leq 2.0$.

For the problem considered here, it is shown that at relatively big values of Re the steady flow tends to be oscillatory unstable. The following section shows the transition from steady to unsteady oscillatory flow. It is known that the typical sequence of evolution of a dynamic system towards chaos for increasing values of the control parameter consists of the following stages: transition to an oscillatory or periodic state; a quasi-periodic regime, and finally chaos (or turbulence). To detect the regime of flow, i.e. transient or steady-state, a series of numerical calculations are performed for each case. Figure 3 shows the temporal evolutions of axial velocity, $\mathrm{v}_{z}$ at the monitoring point $(\mathrm{r}=$ $0.493, \mathrm{z}=0.975$ ). These simulations presented steady-state solutions obtained for the various cases (A, B, and $\mathrm{C}$ ) and the ranges of controlling parameters: the Richardson number $(R i=0$ and $R i=2.0)$, Prandtl number $(\operatorname{Pr}=0.015,0.025$, and 0.032), as shown in Fig 3(a, b, c). The oscillatory aspect of the temporal evolutions of the axial velocity, $\mathrm{v}_{\mathrm{z}}$ at the same monitoring point $(\mathrm{r}=0.493$, $\mathrm{z}=0.975)$ is shown in Fig. $3 \mathrm{~d}$ for $R e=2760, R i=0$ and three aspect ratios $\gamma=1.5,2.0,2.5$. It is seen that the increase of $R e$ enhances the fluid motion while for all aspect ratios $(\gamma=1.5,2.0,2.5)$ the evolutions become time-dependent (oscillatory), a behavior that is more intense for $\gamma=2.5$. Therefore, as Reynolds number is increased, swirl strength increases, and hence the ability of waves to propagate against the flow increases Benjamin (1962). The swirling flows in the hydrodynamic case and for $R=0.9$ will be steady and axisymmetric till the critical Reynolds numbers $\operatorname{Re}_{\mathrm{cr}} \approx 2605,2620$, and 2755 when the oscillatory instability begins to set in, for $\gamma=1.5,2.0$, and $\gamma=2.5$, respectively (see Fig 7). It should be noted that the motivation for the three-dimensional study since the flow can become asymmetric (non-axisymmetric) for certain Reynolds numbers which exceed the transition threshold see (Mahfoud et al. 2016).

The rotating flow developed in the annulus configuration when compared with the flow in a cylindrical container can give a supplementary view into the behavior of the rotating flow near to the inner cylinder, as the conditions at the rotating bottom are different in this case. The inner radii in the annulus configuration have an important role in the capacity of flow absorption. The flow in an annulus can be explained as follows. In the proximity of the rotating bottom disk, the fluid has aspired from the central region near the inner cylinder. Then this fluid is driving in the direction of the outer wall and spirals upwards to the stationary-top disk. Finally, a central core flow swirling in direction of the rotating disk is produced. In the case when the upstream flow is supercritical, while the downstream flow is subcritical, the interaction between these flow regimes is small and they both are completely controlled by appropriate flow conditions. If the interaction between the upstream flow and the downstream flow is small, so the upstream flow completely depends, for a given geometry, on the Reynolds number. The subcritical flow is controlled by the boundary layer at the rotating disk absorbs the fluid driven by the central core. The separation bubble in an annulus is intensified strongly by viscous effects caused by the difference between the flow structures in the central core. Benjamin (1962), suppose the development of standing waves can lead to the appearance of an adverse pressure gradient and, consequently, to the appearance of a stagnation point progressing into a separation bubble.

\subsection{Aspect Ratios and Rotation Rate Effects}

To investigate the effects of the aspect ratios $(\gamma)$ on the behavior of the vortex breakdown, i.e. apparition, location, or suppression of the isothermal cases of $R e=2000$, with increasing the aspect ratios $(\gamma=1.5,2.0$ and 2.5) are considered. 


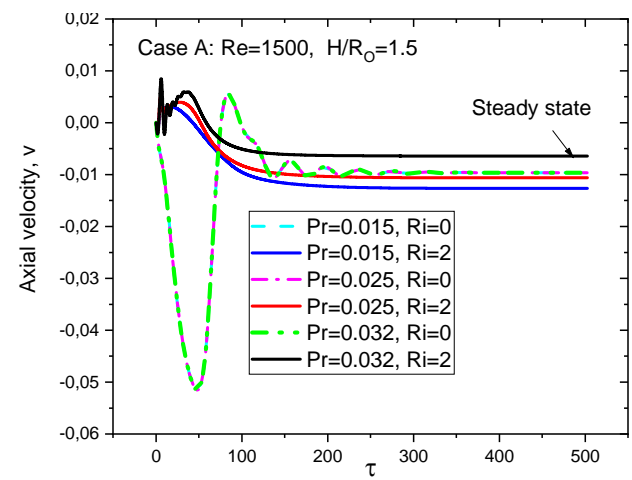

(a)

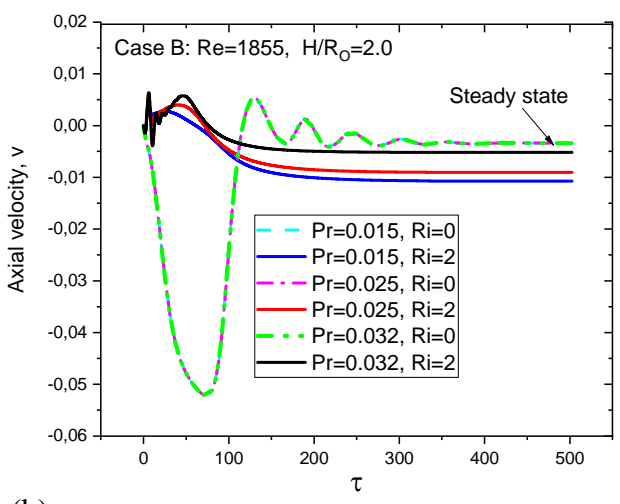

(b)

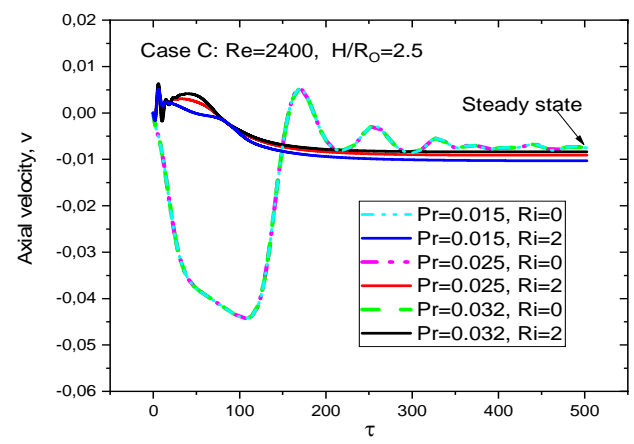

(c)

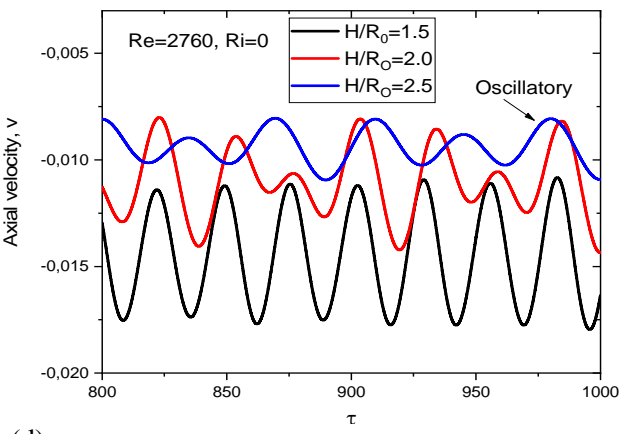

(d)

Fig. 3. Evolution of the velocity, $v$ at the monitoring point $(r=0.493, z=0.975)$.
It is observed, in all cases, that the increase of rotation rate accelerates the fluid and leads to the creation of a vortex breakdown bubble that occurred on the wall of the inner cylinder. The streamlines confirm that only one vortex breakdown exists for $\gamma=1.5$ and 2.0 as shown in Fig.4. However, at $\gamma=2.5$, no vortex breakdown appears, this is observed from the plots of the isolines in Fig. 4. So, a decrease in the size of the vortices is shown, and their position is stuck to the inner cylindrical wall. The central positions of the vortex on the $z$-axis are at $z=1.101$ and 1.395 for $\gamma=1.5$ and 2.0, respectively.On $r$ axis, the central positions are at $r=0.167$ and 0.191 for $\gamma=1.5$ and 2.0 , respectively.

The spatial structure of the zero value of the axial velocity is plotted in the lower of Fig.4. It can shows be observed in the cases of $\gamma=1.5$ and 2.0, that the recirculating bubble plays the role of a solid body and becomes an obstacle to axial flow. The deflected axial flow around the bubble gives a wake region with a low velocity that is formed downstream of the bubble. The viscous and inertia are negligible in the vortex breakdown bubble where the axial velocity is zero. No bubble formed because of no vortex breakdown when $\gamma=2.5$ as shown in Fig. 4. The diameters of the bubbles are 0 . 44 and 0.35 for $\gamma=1.5$ and 2.5 , respectively.

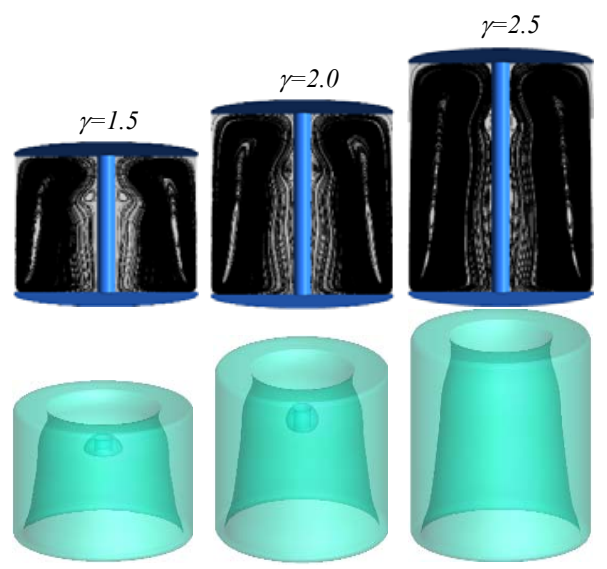

Fig. 4. Iisothermal case $(R i=0)$ of $R e=2000$ with $R=0.9$ at different aspect ratios $H / R_{o}=1.5,2.0$ and 2.5 .

The influence of the intensified rotation rate on the flow pattern, the apparition, and suppression of vortex breakdown is also depicted in Fig. 5 where six Reynolds numbers, $R e=1250,1500,1750,2000$, 2250 , and 2500 , respectively) are compared for one aspect ratio $\gamma=1.5$. When the Reynolds number is increased with an increment of $\mathrm{Re}=250$, the vortex breakdown appears at $R e=1250$ in which, the central position of the vortex on the $\mathrm{z}$-axis is at $\mathrm{z}=$ 0,84 and on the r-axis is 0.16 . Then the size of the vortex grows with the increasing Reynolds number to $R e=1750$. The central positions of the vortex on the $\mathrm{r}$-axis are $|\mathrm{r}|=0,192$ and $|\mathrm{r}|=0.201$ for $R e=1750$ and $R e=2000$, respectively. As clearly shown by the streamlines on the cases of $\mathrm{Re}=2250$ and $\mathrm{Re}=2500$ 

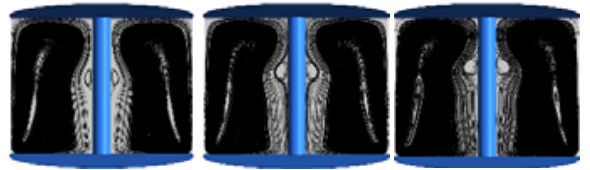

$\operatorname{Re}=1250$

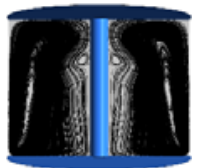

$\mathrm{Re}=2000$
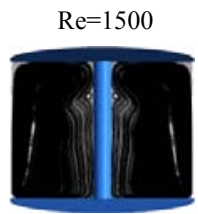

$\operatorname{Re}=2250$
$\mathrm{Re}=1750$

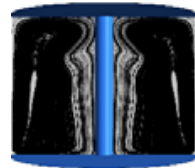

$\mathrm{Re}=2500$

Fig. 5. Iso-contours of the stream function in case of $\gamma=1.5$ for $R=0.9$ at different Reynolds numbers, $\boldsymbol{R e}$.

that a small vortex appears in these two cases, and disappears for $\mathrm{Re} \geq 2510$.

Figure 6 shows the effects of increasing Reynolds numbers on the central position of the vortex on the $z$-axis and $r$-axis, respectively in the case of $\gamma=1.5$. Here, the central position is presented at different Reynolds numbers, which is in the range of $1100 \leq$ $R e \leq 2500$. First, a vortex appears at $R e=1170$ and disappears at $R e \approx 2510$. The central position on the $z$-axis is at $z=0.780$ when the small vortex appears at $R e=1175$ and rise to $z=1.157$ until $R e=2500$. The central positions on the $\mathrm{z}$-axis of the vortex increase gradually with the increasing Reynolds number. The red curve with circle symbols shows the central position of the vortex on the $r$-axis which is different from those in the central position on the z-axis. In this case, the distribution of the central position on the $r$-axis represents the shape of a semicircle. The central positions on the $r$-axis are divided into two parts, when $R e \leq 1800$, the r-central position of vortex increase with the increasing $R e$, contrarily when $R e \geq 1800$, they decrease with the increasing $R e$. Finally, for the case of $\gamma=1.5$, the present results show that the increase of the $R e$ causes the increase of the z-central position of the vortex, but contrarily causes the decrease of the rcentral position after it passes a peak. The situation can be interpreted as follows, the peak occurred in the region where the viscous and inertial forces are of the same magnitude. Moreover, Based upon experiments of Escudier (1984), the breakdown region is characterized by a radius $r$, the corresponding Reynolds number is then $(r /$ $\left.R_{0}\right) R e^{1 / 2}$. The largest value of this quantity for the case $\gamma=1.5$ is for $\mathrm{Re}=1800$ at $r / R_{o} \sim 0.202$ and $\left(r / R_{o}\right) R e^{1 / 2} \sim 8.57$.

For $R=0.9$, the boundaries (limits zones) of the vortex breakdown of the present results are compared with the results from Escudier's stability diagram for a cylindrical container $(R=1.0)$, see Fig. 7. Escudier (1984) performed an experimental study using a laser-induced fluorescence technique to visualize the flow structure in cylindrical geometry generated by the rotating bottom disk. They have proposed a stability diagram describing the vortex breakdown regime which corresponds to the black curve in Fig. 7. The aspect ratios effect on

the vortex breakdown zones in the $(R e, \gamma)$ plane shows how the boundaries of the vortex breakdown shift towards the left, i.e. the vortex breakdown zone are moved to the weak values of aspect ratios for the annular gap $(R=0.9)$ which correspond to red curve wit stars symbols in Fig. 7 . The intersection point between these two curves becomes at the aspect ratio $(\gamma)=1.2$ close to $R e \approx 1015$. On the other hand, the increasing effects of the Reynolds number rises also the transition to an unsteady regime for $R=0.9$.

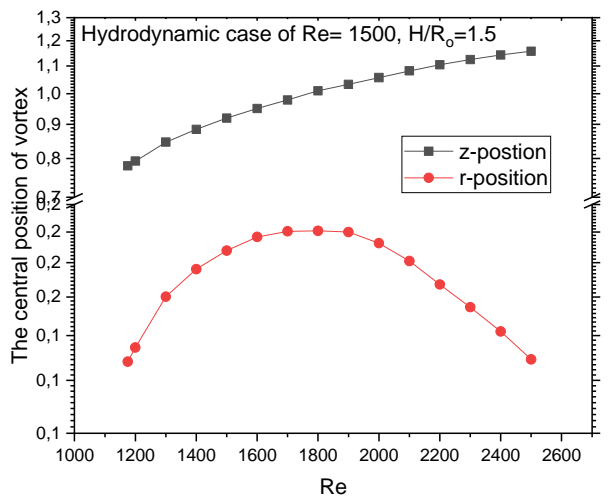

Fig. 6. Central position of the vortex on the $\mathrm{z}$ axis and r-axis vs $R e$

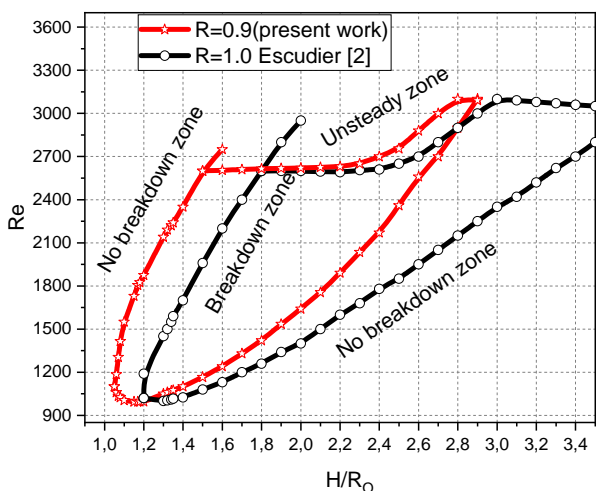

Fig. 7. Boundaries of the vortex breakdown zone of the present results $(R=0.9)$ compared to the limits taken from Escudier (1984) for $R=1.0$.

\subsection{Buoyancy Effect on Vortex Breakdown and the Fluid Layers}

\subsubsection{Case of $\mathrm{Pr}=0.015$}

To investigate the effects of thermal gradients on the fluid layers and the control of the vortex breakdown (i.e. location, or suppression of vortex breakdown), taking the case of liquid aluminum, corresponding to $P r=0.015$. One axisymmetric bubble is attached to the inner cylinder as shown in Fig. 8 for case $\mathrm{A}$ at $R i=0.01$ corresponding to $R e$ $=1500$ and $\gamma=1.5$. The almost isothermal case, in which forced convection takes place. The rotation of the bottom gives a centrifugal force to the fluid, and this force drives the fluid radially outward. The 
upward jets and downward flow result in the centrifugal flow structure, with an axial vortex near the inner cylinder axis. The central position on the $\mathrm{z}$-axis of the vortex breakdown is at $\mathrm{z}=0.91$, and on the $\mathrm{r}$-axis is at $|\mathrm{r}|=0.19$, respectively. It is observed for $R i=0.01$ the domination of the convection mode of heat transfer, where a remarkable deviation is shown in contour plots of isotherms(Fig.8). However, the buoyancy forces adverse pressure gradient, which tends to suppress the non-homogeneities, and then it can be expected disappearance of the vortex breakdown when $R i=0.1$. In this case, the core rotates at an exact average velocity between that the two end disks, which requires that the core will be in azimuthal equilibrium between the viscous stress from the rotating bottom disk and the viscous decelerating at the stationary top disk. When the $R i$ is increased further to $R i=1.0$, the buoyancy forces reacting on the fluid near the top hot disk are very intense that the inertia forces result from the rotation of the bottom disk cannot tend the fluid downward and the flow is structured now with two-cell, which has a smaller size (Fig. 8c). As the Richardson number is increased from $R i=1.5$ to 2 , the buoyancy force acting on the hot fluid near the top disk, which produces another region (counter-rotating flow). This region grows in size as the $R i$ is increased more until it takes the entire top section of the annular gap $(R i=2$, Fig. 8e). So the increase of $R i$ results in a damping of the fluid motion, and thus heat conduction progressively dominates over convection heat transfer when $\mathrm{z}>1$.

In the second line of Fig. 8 the effect of $R i$ is investigated for the case of $R e=1855$ and $\gamma=2.0$ (case B). For $R i=0.0,1$ a small breakdown bubble compared to case (A) can be seen in Fig. 8a, centered on the $\mathrm{z}$-axis at $\mathrm{z}=1.34$ and $\mathrm{r}$-axis at $|\mathrm{r}|$ $=0.71$, respectively. As is clearly shown in contour plots of isotherms when $R i=0.01$, convective heat transfer dominates the temperature distribution. A short increase in the Richardson number to $R i=0.1$ (Fig. 8b) causes the disappearance of the vortex breakdown bubble. Another cell becomes apparent when $R i=1.0$, due to the counter-rotating flow. This is depicted in Fig. 8c, where the streamlines show that a double-layer flow structure occurs. When $R i$ is increased further ( $R i=1.5$ to 2$)$, causes the growth of regions of counter-rotating flow near the hot disk.

In the third line of Fig.8, we also give the effect of Richardson's number for the case of $\operatorname{Re}=2400$ and $\gamma$ $=2.5$ (case $\mathrm{C}$ ). In $R i=0.01$ case, a one-bubble vortex breakdown appears and is centered at $\mathrm{z}=1.81$, and $|r|=0.14$. The observation is almost the same as the previous case when $R i=0.1$ (Fig 8b), the vortex breakdown is suppressed, and the fluid in the bulk of the annular gap exhibits quasi-rigid rotation. A counter-rotating flow zone becomes apparent when $R i=1.0$ near the hot top disk. Increasing the Richardson number to $R i=1.5$ leads to the appearance of a stratified structure with three fluid layers. Also, one clockwise recirculation region appears between two counterclockwise recirculation regions when $R i=2$, which has a double lobe structure. As is clearly shown by the isotherms in Fig.8e, the vertical temperature gradient is concentrated in the vicinity of the top boundaries where the conduction dominates the thermal transport. This is a consequence of the thermal insulation by the stratified layers.
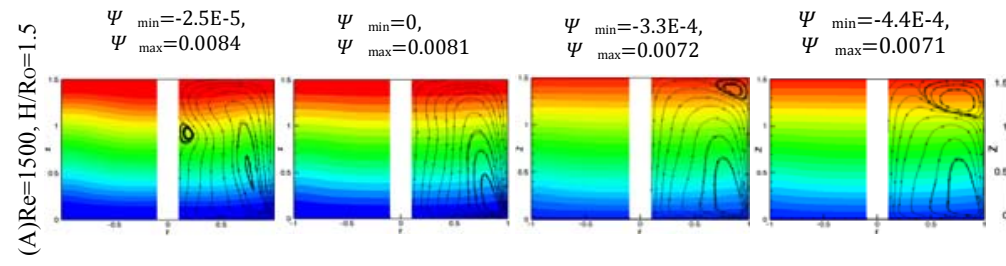

$\Psi_{\min }=-5.3 \mathrm{E}-$ 4 ,
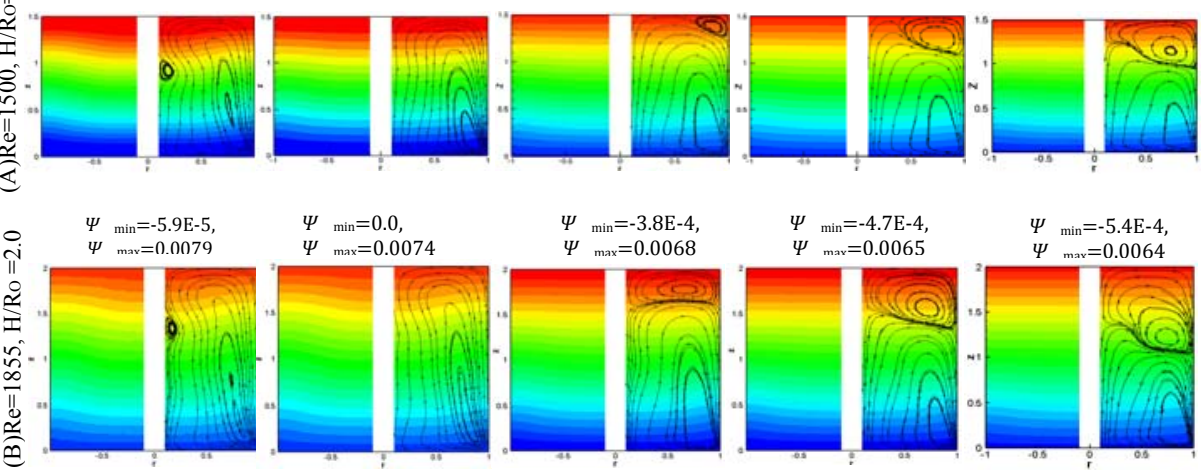

$\Psi_{\min }=0.0$,
${ }^{\prime}=0.0074$
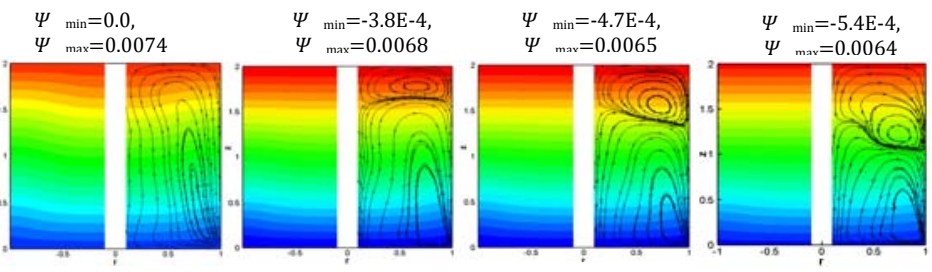

$\psi_{\min }=-6.1 \mathrm{E}-5$
${ }_{\max }=0.0072$

$\Psi \min =0.0$,

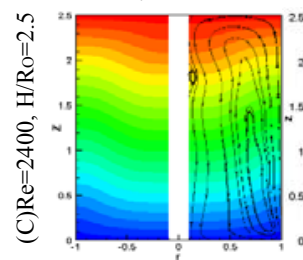

(a) $R i=0.01$

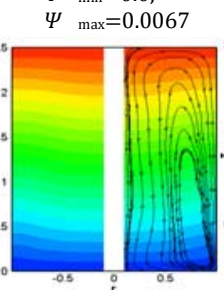

(b) $R i=0.1$

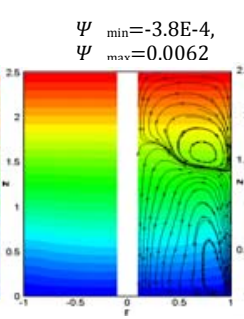

(c) $R i=1.0$

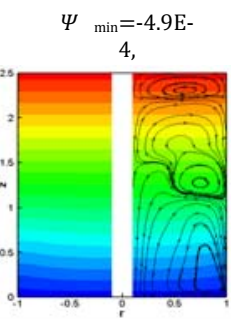

(d) $R i=1.5$
$\Psi_{\min }=-5.8 \mathrm{E}-4$, $\Psi_{\max }=0.0057$

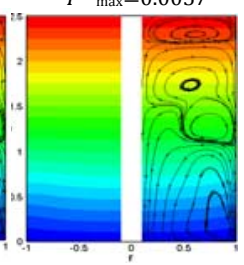

(e) $R i=2.0$

Fig. 8. Superposed streamlines and isotherms in meridional planes for increasing $\operatorname{Ri}$ when $\operatorname{Pr}=0.015$. 


\subsubsection{Case of $\operatorname{Pr}=0.025$}

Figure 9 presents the isolines of stream function for progressively increasing Richardson numbers when $P r=0.025$. The structure of the flow in Case A is almost the same as case A when $\operatorname{Pr}=0.015$ as mentioned above. Similar to what was observed in the previous section, a further increase in $R i$ causes the vortex breakdown bubble to disappear at $R i=$ 0.1 , see case A (Fig. 9-b). When the Richardson number is increased to $R i=1.0$, the buoyancy forces induce a clockwise recirculation region that appears in the upper annular gap (Fig. 9-c). As in the previous case, further increases in $R i$ cause this new fluid region to occupy almost the upper half-annular gap; however, in this case, the fluid recirculation is highest and then decreases quickly with the increasing $R i$.

In the second line of Fig. 9, the case of $R i=0.1$ is almost similar to what was observed in the previous section, we also noticed here when $R i=0.1$ the vortex breakdown bubble has disappeared (Fig. 9c). The transition to a double layer flow structure is observed at $R i=1.0$, in which a new clockwise region has formed near the stationary top disk. There are further transitions that occur, when $R i$ further increases beyond 2.0, leading to the formation of three fluid layers. Therefore, more increases in the $R i$ lead to a gradual reduction in the stream function.
As to case $\mathrm{C}$, the effects of the buoyancy forces on the transition to a multi-layered flow pattern are the strongest among these cases. The one-bubble vortex breakdown centered near to inner cylinder wall $(R i$ $=0.01$ ) disappears in the interior of the container at $R i=0.1$ (plots in (C) of Fig. 9b). When the temperature stratification is important $(R i=1.0)$, while the Ekmàn suction disappears in the interior of the gap and leads to the formation of additional layers (plot in $(\mathrm{C})$ of Fig. 9c). Then the number of layers grows with the increasing Richardson number, and it is four layers when $R i=1.5$. When $R i=2.0$ it reaches the peak, which is five layers, but not all of the formed layers occupied all the distance from the sidewall to the inner cylinder wall.

A remarkable deviation is shown in contour plots of isotherms when $R i \leq 0.1$ in the all annular gap area, where convective heat transfer dominates the temperature distribution. This behavior is in contrast to $R i \geq 1$ cases in which the domination of the conductive heat transfer can be observed a remarkable deviation is shown in contour plots of isotherms when $R i \leq 0.1$ in the all annular gap area, where convective heat transfer dominates the temperature distribution. This behavior is in contrast to $R i \geq 1$ cases in which the domination of the conductive heat transfer mode can be observed in the upper annular gap, due to the insulation created by the layers of which are developed.
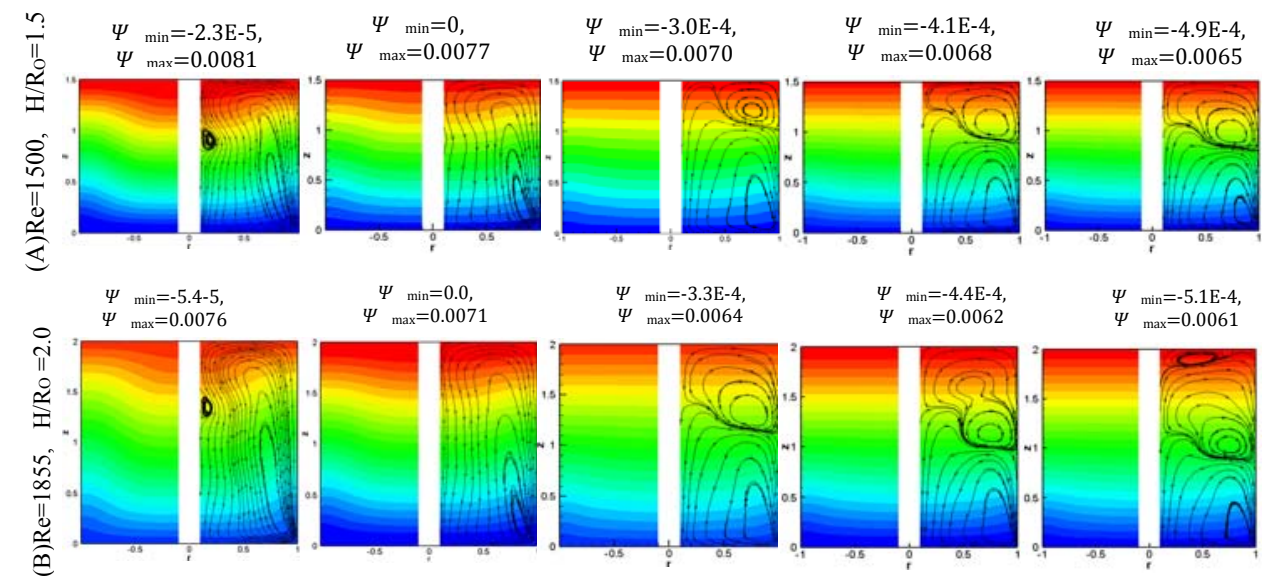

$\Psi_{\min }=-4.4 \mathrm{E}-4$,

$\Psi \quad{ }_{\max }=0.0062$

$\Psi_{\min }^{\max }=-5.1 \mathrm{E}-4$,
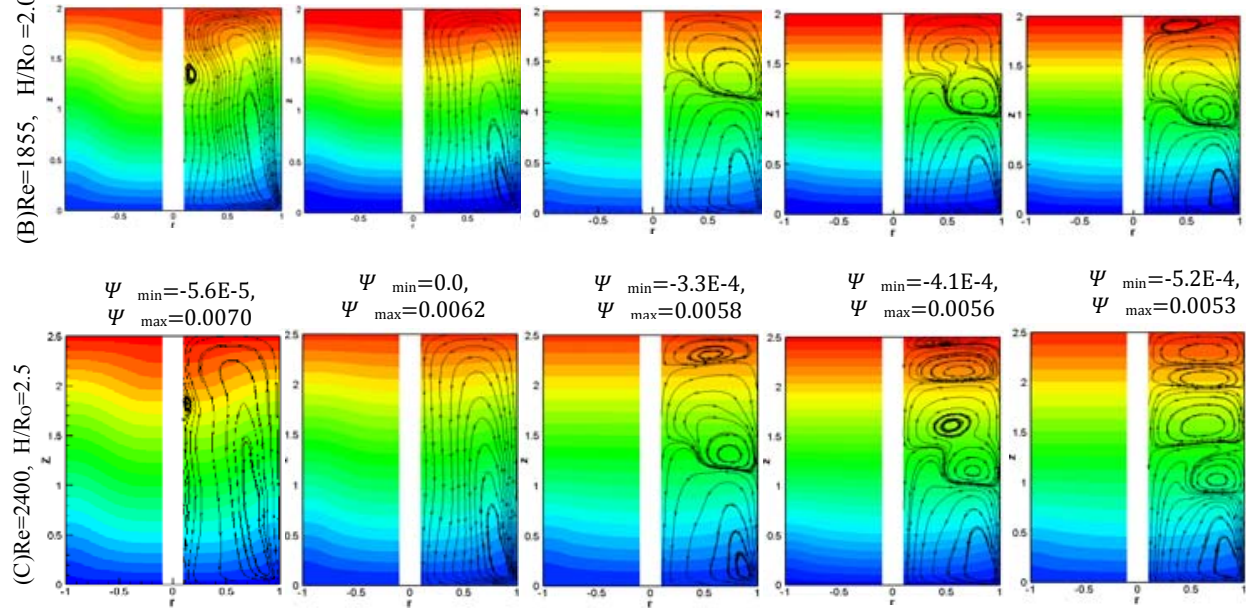

$\Psi \min =0.0$,

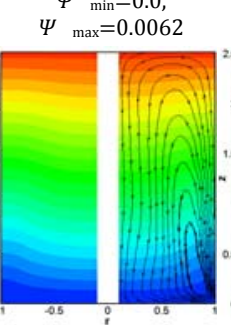

$\Psi \quad \min =-3.3 \mathrm{E}-4$,
$\Psi \quad \max =0.0058$

$\Psi_{\min }=-4.1 \mathrm{E}-4$,
$\Psi_{\max }=0.0056$

$\Psi \min =-5.2 \mathrm{E}-4$
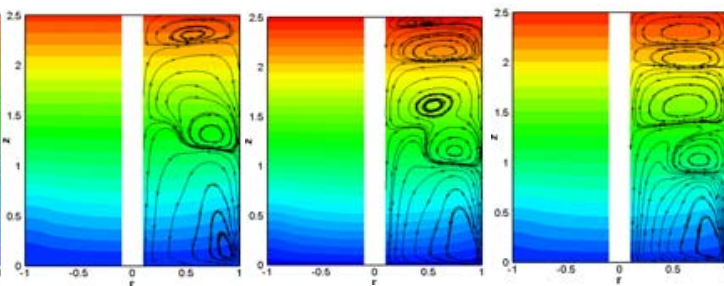

(a) $R i=0.01$

(b) $R i=0.1$

(c) $R i=1.0$

(d) $R i=1.5$

(e) $R i=2.0$

Fig. 9. Superposed streamlines and isotherms in meridional planes for progressively increasing Richardson numbers when $\mathrm{Pr}=0.025$. 


\subsubsection{Case of $\operatorname{Pr}=0.032$}

Figure 10 presents the superposed streamlines and isotherms in meridional planes for progressively increasing Richardson numbers when $P r=0.032$.

The results for case (A) show that, when the Richardson number increased to $R i=0.1$, the vortex breakdown is suppressed. In this case, heat transfer convection is dominant. The maximum value of the stream function decrease with increasing $R i$ until 0.0064 at $R i=2.0$. The streamlines when $R i=1.0$ show a new region of counter-flow which grows with increasing $R i$ and then dominate the entire top section of the annular gap (plot in A of Fig. 10).

The plots in Fig. 10 for case (B) show the disappearance of the vortex at $R i=0.1$ and then the decomposition in the counter-flow region, up to two-layered appear for $R i=1.0$ (plot in A of Fig. $10 \mathrm{c})$. The stratified structure with four fluid layers is observed when $R i=2.0$. Note that, the separated zone centered at $\mathrm{z}=0.90$, and $|\mathrm{r}|=0.75$ does not occupy the entire distance from the outer wall to the inner wall. The isotherms plot when $R i=2.0$ shows that conduction mode dominated the heat transfer, especially in the top region.

Similarly, the buoyancy acts for case $\mathrm{C}$ are stronger than those in cases A and B (plots in C of Fig.10).

When the vertical temperature gradient is small $(R i=0.1)$, and the convection mode dominates heat flux, the vortex breakdown in the annular gap is suppressed. In the range of $R l \geq 1$, there is no big difference about the isothermal line distribution, but the maximum value of non-dimensional streamlines $\left(\Psi_{\max }\right)$ decreases as the increasing of Richardson number, and are 0.0065 and 0.0055 at $R i=0.1$ and $R i=2$, respectively, that indicates the flow is suppressed by the Buoyancy force. The process of increasing the Richardson number to $R i=1.0$ induces three layers (plots in $\mathrm{C}$ of Fig. 10c). On the range of $R i$ considered in the case (C), up to five layered appear for $R i=1.5$ ( see, Fig.10d) and six such layers are observed for $R i=2.0$ (Fig.10e).

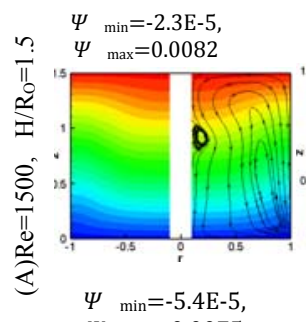

$\psi_{\text {min }}=0.0$ $\Psi \quad \max =0.0074$

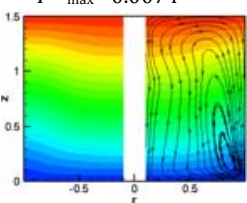

$\Psi \min =0.0$,
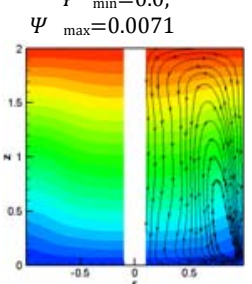

$\Psi_{\min }=0.0$

$\Psi_{\max }=0.0066$

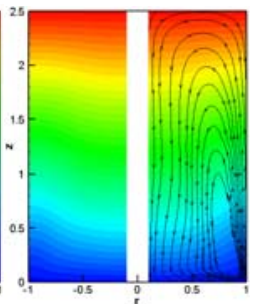

(b) $R i=0.1$

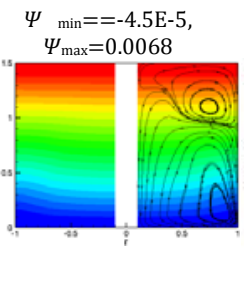

$\Psi_{\min }=-4.0 \mathrm{E}-4$,

$\Psi \quad{ }_{\max }=0.0063$

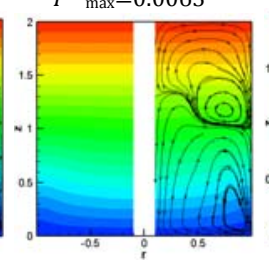

$\Psi_{\min }=-4.1 \mathrm{E}-4$,

$\Psi \quad{ }_{\max }=0.0058$

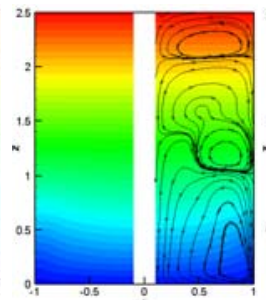

(c) $R i=1.0$ $\psi_{\min }=-2.6 \mathrm{E}-4$ $\Psi_{\max }=0.0066$

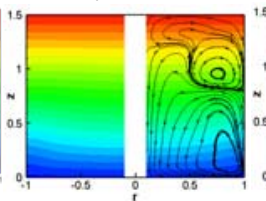

$\Psi_{\min }=-4.6 \mathrm{E}-5$,

$\Psi_{\max }=0.0061$

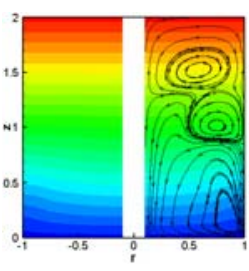

$\psi_{\min }=-5.3 \mathrm{E}-4$

$\Psi \quad{ }_{\max }=0.0055$

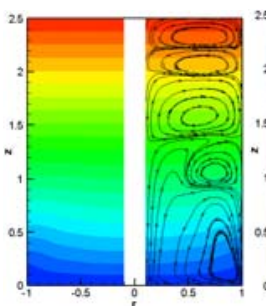

(d) $R i=1.5$
$\Psi \min =-5.7 \mathrm{E}-4$

$\Psi{ }_{\max }=0.0064$

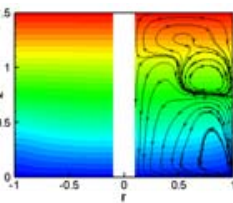

$\Psi \min =-6.1 \mathrm{E}-4$

$\Psi \quad \max =0.0059$

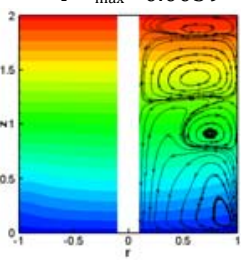

$\Psi \min =-6.4 \mathrm{E}-4$,

$\psi \psi_{\max }=0.0053$

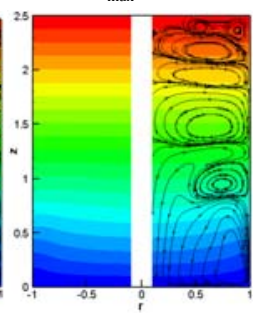

(e) $R i=2.0$

Fig. 10. Superposed streamlines and isotherms in meridional planes for progressively increasing Richardson numbers when $\mathrm{Pr}=0.032$.

Figure 11a shows the evolution of the number of fluid layers for the case of $P r=0.015$ and for A, $\mathrm{B}$, and $\mathrm{C}$ cases, which are discussed above. The number of layers is the number of stratified recirculation zones in the meridional flow. Here, the flow is characterized by one concentrated vortex when $R i=0.01$, this vortex undergoes breakdown, i.e. a stagnation point followed by a small recirculation zone near the inner cylinder wall. The flow contains a single layer, that occurs for $R i \leq 0.3$ (case A). It was found also that the number of layers, corresponding to case A, grows with the increase of Richardson number and leads to a 
second layer to form beyond $R i>0.3$. As to case $\mathrm{B}$, a second layer flow structure is observed when $R i \geq$ 0.5 . In case (C), up to three layers appear for $R i \geq$ 1.3 .

The evolution of the number of fluid layers for the case of $P r=0.025$ is summarized in Fig. $11 \mathrm{~b}$. Similar to the case of $P r=0.015$, the number of layers is computed by the number of recirculation zones occupying a distinct part of the meridian flow. It is clear that for case $\mathrm{A}$, the flow contains a single layer, that occurs for $R i \leq 0.6$. Then, more increases in the Richardson number beyond $R i \geq 0.7$, can be expected to lead to a second layer to form. As to case B, the effects in the growth of Richardson's number on the evolution of stratified layers are stronger than in case A. Here (case B), a three-layer flow structure is observed when $R i \geq 1.6$. On the range of $R i$ considered in the case $(\mathrm{C})$, up to four layers appear for the range of $1.4 \leq R i<1.7$ and five such layers are for $R i \geq 1.7$.

The evolution of the number of fluid layers for the case of $P r=0.032$ is summarized in Fig. 8c. It is observed that the effect of vertical temperature difference on the flow pattern is to lead the meridional flow into layered structures. It is clear that for case A, the flow contains a single layer, that occurs for $R i \leq 0.6$. Then, more increases in the Richardson number beyond $R i \geq 0.7$, can be expected for a generation of a second layer to form. As for case B, a three-layers are observed for $R i \geq 1.3$. Then four fluid layers are formed in the range of $1.8 \leq R i \leq 2$. For case (C), up to four layers appear for $1.3 \leq R i \leq 1.4$, and then five layers appear for $R i=1.5$ to $R i=1.8$ and six layers are observed for $1.9 \leq R i \leq 2$.

Figure 11d compares the evolution of the number of fluid layers for the three Prandtl numbers $(P r=0.015,0.025$, and 0.032 , respectively) when $R e=2400$ and $\gamma=2.5$ (case C). Figure 11d indicates that Prandtl number $P r$ has a major influence on the number of fluid layers formed. The concurrence between buoyancy and viscous forces is amplified with increasing $R i$, therefore the number of fluid layers increases with increasing $R i$ in all investigated cases. Therefore, the number of formed layers in the case of $P r=0.032$ is bigger than in other cases $(\operatorname{Pr}=0.015$ and 0.025$)$.

\subsection{Heat Transfer}

We believe that the relationship between the stratification of fluid and heat transfer must be clarified. However, the Nusselt number is analyzed in the remainder of this section for many parameters. The decreasing of the average Nusselt number with the Richardson number is presented in Fig. 12a, where three combinations (A, B, and C) are compared at $P r=0.032$. Figure 12a shows that $\overline{N u}$ monotonically decreases with increasing $R i$ and approaches the reciprocal of the aspect ratio, i.e., $1 / H(=0.4$ at $\gamma=1.5 ; 0.5$ at $\gamma=2.0$ and 0.66 at $\gamma=1.5)$ which means the value of the conduction limit. In Fig. 12a, it is noticed that the maximum value of $\overline{N u}$ is attained when a single layer fluid is established in the meridian flow. For mixed convection, the influence of buoyancy.

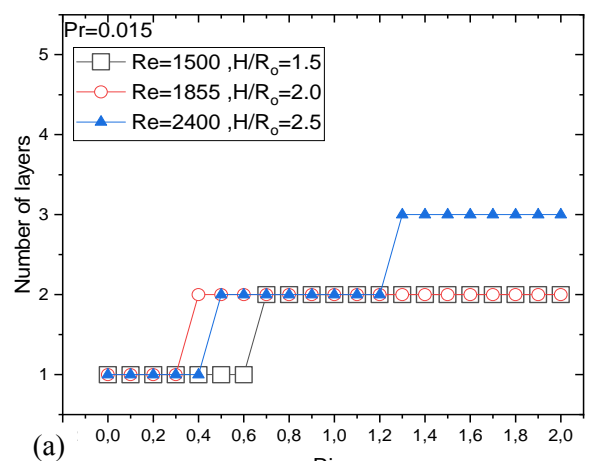

(a) $\quad \begin{array}{lllll}0,0 & 0,2 & 0,4 & 0,6 & 0,8\end{array}$

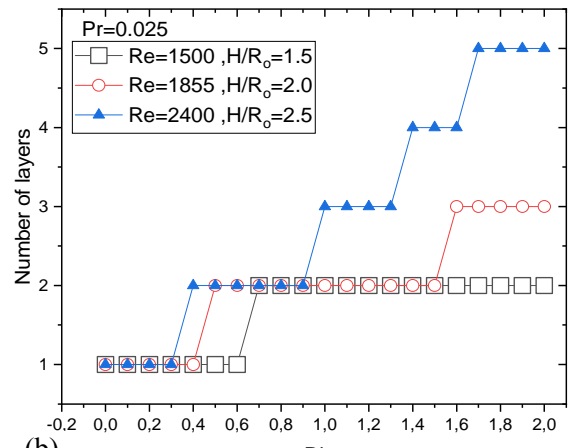

(b)

$\mathrm{Ri}$

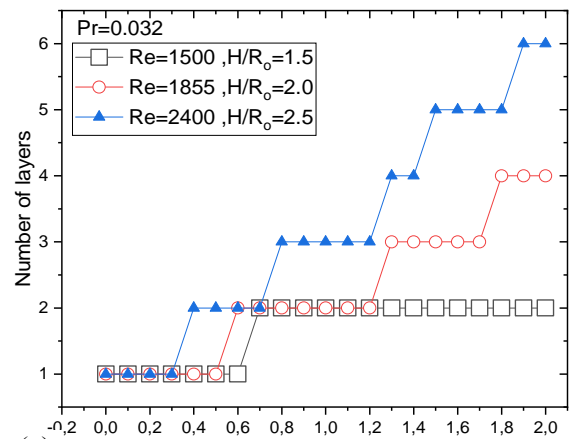

(c)

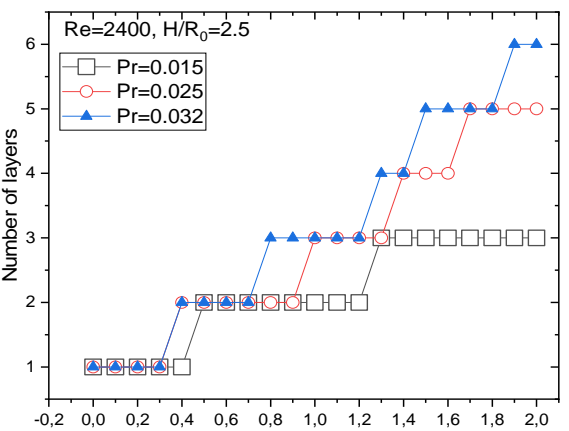

(d)

$\mathrm{Ri}$

Fig. 11 Effect of Richardson number on the number of flow stratification layers formed.

For mixed convection, the influence of buoyancy force becomes stronger with the rising of the Richardson number, so the concurrence between 
viscous and buoyancy forces is increasingly important with increasing Ri. However, the lighter hot fluid close to the top hot disk sits on the top of the heavier cold fluid close to the bottom cold disk. The effects of natural convection continue to exist only near the heated top disk. In this case, the stable stratification of fluid opposes the flow produced by the bottom rotating disk, and so the net advective transport diminishes, and the value of $\overline{N u}$ decreases with increasing Ri. Also, these fluid layers play the role of thermal insulation, since the number of layers influences the heat transfer. We conclude that the combination of $R e$ and $\gamma$ control the heat transfer by the presence or absence of fluid layering.

Figure $12 \mathrm{~b}$ shows the decrease of $\overline{N u}$ with progressively increasing $R i$ for different $P r$ at case A $(R e=1500$ and $\gamma=1.5)$. For all values of the Prandtl number studied here, the average Nusselt becomes insensitive to the changes when the Richardson number goes beyond 1.2. For case (A) it appears that $\overline{N u}$ reaches extreme value when $R i=0$ and is $0.75,0.78$, and 0.86 for $P r=0.015,0.025$, and 0.032, respectively (Fig.12b). Furthermore, it is seen that at a constant value of $R i$ the average Nusselt values become progressively grow as the Prandtl number is increased indicating that advective transport reinforces with increasing $\mathrm{Pr}$, as observed from Fig. 12b. Conscontely Prandtl number $\mathrm{Pr}$ has an important influence on $\overline{N u}$ in this case.

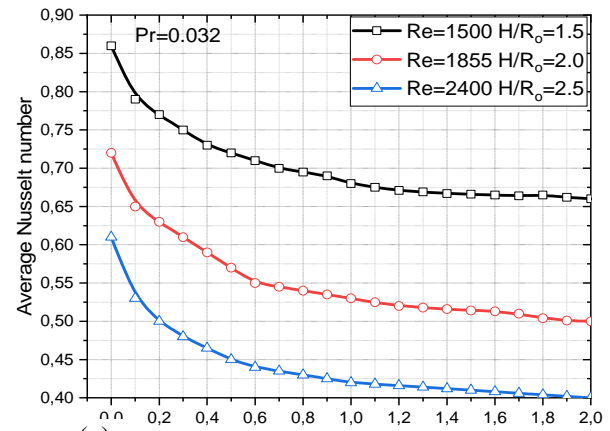

(a)

$\mathrm{Ri}$

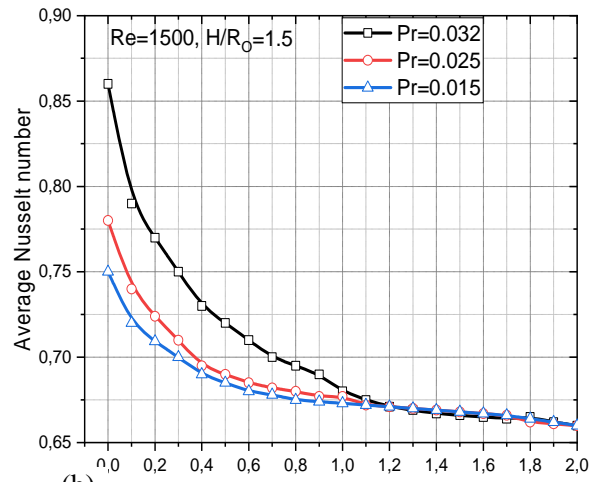

(b)

Fig. 12. Effect of the Richardson number on the average Nusselt number.

\subsection{Magnetic Effect on Fluid Layers}

Under magnetic effect, viscous forces can complete with magnetic forces in the Hartmann layer near to walls normal the applied magnetic field. Thus, when the Hartmann number increases, the Ekman layer is progressively replaced by the Hartmann layer, which is near to walls normal to the magnetic field. These layers are perpendicular to the field, having a dimensionless thickness $\delta \perp \approx 1 / \mathrm{Ha}$. Therefore, the intensified magnetic field results in Hartmann layer thickness. Where the walls are electrically insulating, the Hartmann layer near the disk has a similar solution as in the case of the classical Ekman layer. The order of magnitude of the axial velocity is $\mathrm{v}_{z} \sim \operatorname{Re} / \mathrm{Ha}^{3}$, which results in the weakness of the axial velocity.

To investigate the effects of the axial magnetic field on the vortex breakdown in hydrodynamic case taking, for example, the case of $\mathrm{Re}=1500$ and $\gamma=1.5$ for progressively increasing Hartmann numbers, $H a=1,5,7$, and 9, respectively. Figure 13, shows that breakdown size diminishes with increasing Hartmann numbers and finally disappears at $H a=9$.

The magnetic effect on the layering (i.e., apparition or suppression) is shown in the case of $\operatorname{Pr}=0.032$ and $R i=1.0$ in the three cases mentioned above. The streamlines plots in the case of $R i=1.0$ at $H a=0$ show a double layer, in which the bottom layer is the biggest (Fig.14a). The magnetic field in the vertical direction has a good suppressive effect on both vortex breakdown and fluid layers which are shown in this case. As clearly shown by the streamlines on the first line of Fig. 14, the clockwise recirculation top region diminishes in size and moves toward the sidewall when $H a=10$. On the contrary, the counterclockwise recirculation zone grows in size until it takes the entire top gap of the cylinder. Also, the maximum streamfunction decreases with increasing $\mathrm{Ha}$ and is 0.0065 and 0.0061 for $H a=0$ and $H a=10$, respectively. The small toroidal vortex decreases in size and then disappears at $\mathrm{Ha}_{\mathrm{cr}}=20$. The $\mathrm{r}$-central position of the small toroidal region rises with increasing $H a$ and, on the contrary, the z-central diminishes slightly. The central positions are at $\mathrm{z}=1.08,0.99$, and 0.97 for $H a=5,10$ and 15, respectively.

As for case B shown in Fig 14, the streamline plots for $H a=0$, show a double layer, in which the top layer, has a double lobe structure. The length along the z-axis of the clockwise toroidal vortex above $\mathrm{z}=1$ becomes narrow and is about 0.38 at $H a=10$. The streamlines for $H a=15$ and $H a=20$ clearly show the small toroidal vortex centered at $\mathrm{z}=1.1$ and $\mathrm{z}=1.04$, respectively that decreases in size further and disappear at $\mathrm{Ha}=21$.

The effect of increasing $\mathrm{Ha}$ is distinctly seen in Fig. 14 at case $\mathrm{C}$. For $H a=0$, three layers are observed, but at $H a=10$ the middle clockwise toroidal vortex divides into two cells and drives to the creation of three layers. For $H a=20$ the streamlines show a small toroidal vortex centered at $\mathrm{z}=1$, then decreases in size and disappears at $H a_{\mathrm{cr}}=26$. For all 
cases the isotherms plots when $R i=1.0$ show that conduction domine the heat transfer, especially in the top gap.

Figure 15 shows the isocontours in the meridional planes of axial velocity, flowed by the spatial structure of the zero axial velocity plotted for increasing Hartmann numbers for case $\mathrm{C}$ when $\mathrm{Pr}=$ 0.032 and $R i=1.0$. This figure shows the connection between the zero value of axial velocity and the number of formed layers, on the one hand, the relationship between zero axial velocity and the increasing Hartmann number. Therefore, as the Hartmann number increases, the decrease in axial velocity accelerates and sequential flow axial stagnation could occur near the inner cylinder leading to the elimination of fluid stratification. The number of the fluid layers can be approximated from the zero axial velocity. For critical value $H a_{\mathrm{cr}}=26$, the deceleration in axial velocity causes the fluid to stagnated axially near the outer cylinder, and therefore there only one layer is formed.

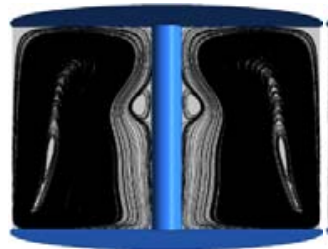

$\mathrm{Ha}=1$

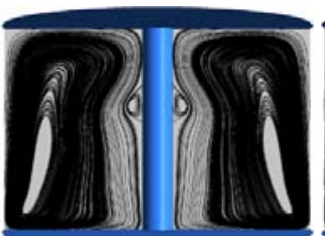

$\mathrm{Ha}=5$

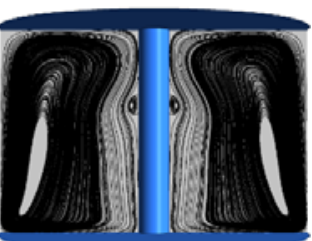

$\mathrm{Ha}=7$

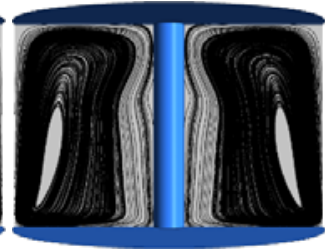

$\mathrm{Ha}=9$

Fig. 13. streamlines in the meridional planes in case of $\operatorname{Re}=1500$ and $\gamma=1.5$ for progressively increasing Hartmann numbers $(\mathrm{Ha}=1,5,7,9)$.

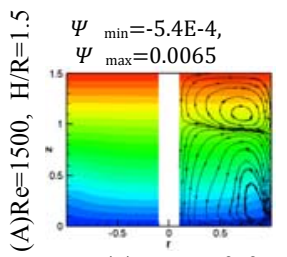

(a) $H a=0.0$

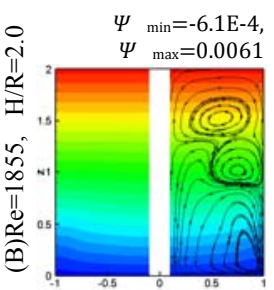

(a) $H a=0.0$

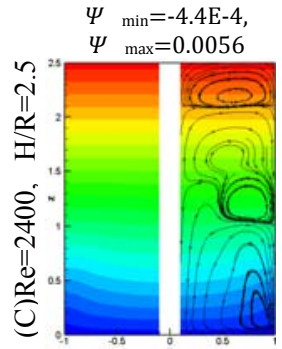

(a) $H a=0.0$

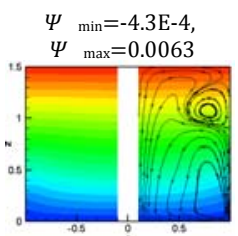

(b) $H a=5$

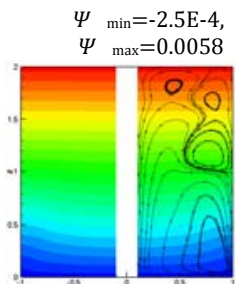

(b) $H a=5$

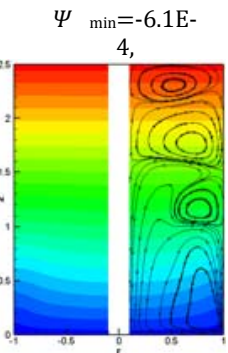

(b) $H a=5$

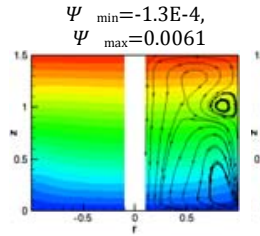

(c) $H a=10$

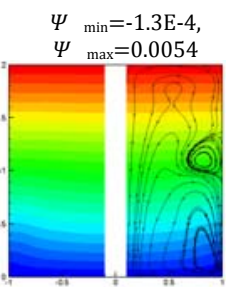

(c) $H a=10$

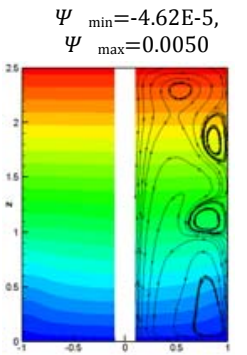

(c) $H a=10$

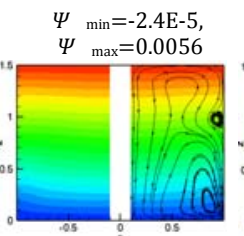

(d) $\mathrm{Ha}=15$

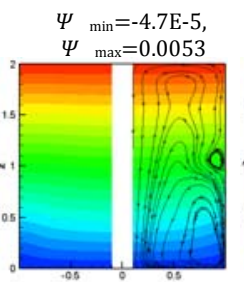

(d) $H a=20$

$$
\Psi \min =-2.62 \mathrm{E}-5 \text {, }
$$$$
\Psi{ }_{\max }=0.0046
$$

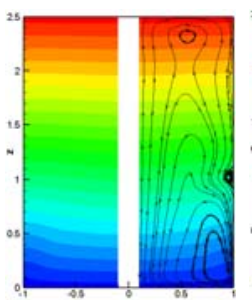

(d) $H a=20$ $\psi$ min $=0$ $\Psi \max =0.0053$

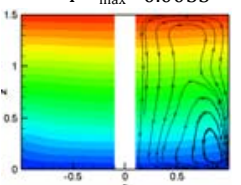

(e) $H a=20$

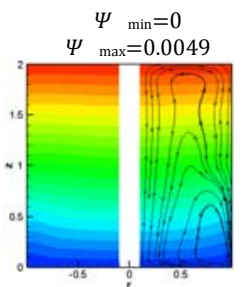

(e) $H a=21$

$\psi \min =0$ $\Psi \quad \max =0.0043$

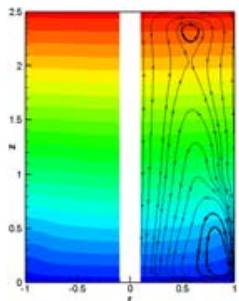

(e) $H a=26$

Fig. 14. Superposed streamlines and isotherms for increasing $\mathrm{Ha}$ when $\operatorname{Pr}=0.032$ and $R i=1.0$. 


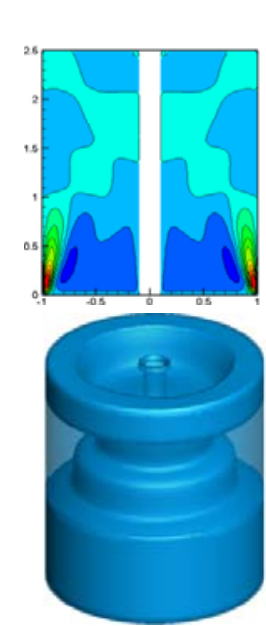

(a) $H a=0.0$

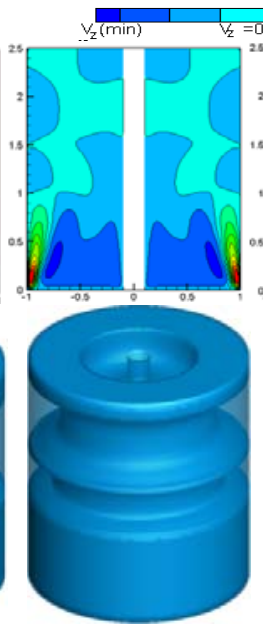

(b) $H a=5$

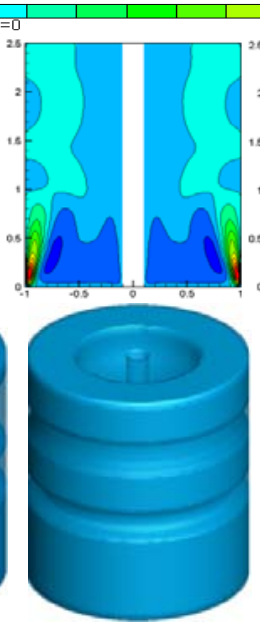

(c) $H a=10$

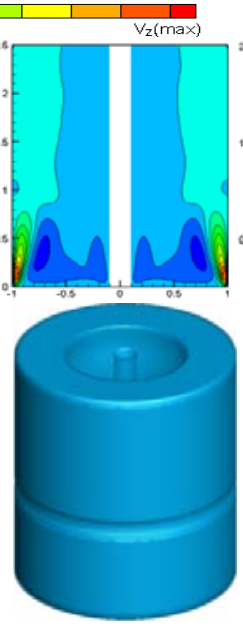

(d) $H a=20$

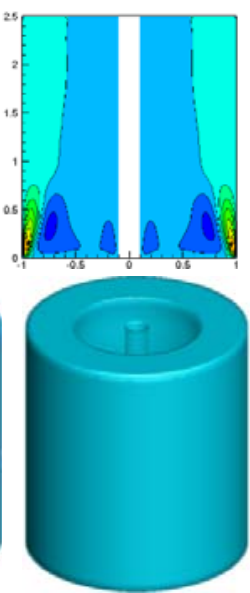

(e) $H a=26$

Fig. 15. Iso-contours of axial velocity in the meridian plane ( top) flowed by the spatial structure of zero axial velocity(bottom) for increasing $\mathrm{Ha}$ when $\mathrm{Pr}=0.032$ and $\mathrm{Ri}=1.0$ at case $\mathrm{C}$.

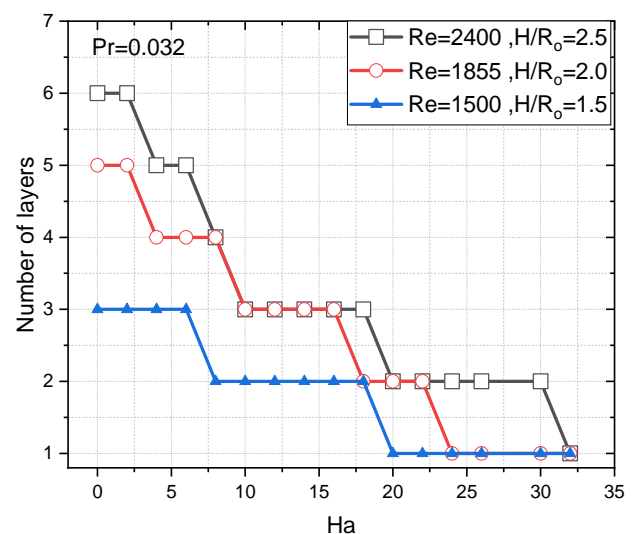

Fig. 16. Reduction of the number of fluid layers VS $\mathrm{Ha}$ when $\mathrm{Ri}=\mathbf{2 . 0}$ and $\mathrm{Pr}=0.032$.

Figure 16 compares the magnetic field effect on the number of fluid layers for three cases (A, B, and C) mentioned above when $P r=0.032$ and $R i=2.0$. The decrease in the curves as shown in Fig.13 indicates that increasing $\mathrm{Ha}$ has an important influence on the number of fluid layers formed. Therefore, the number of fluid layers decreases with increasing $\mathrm{Ha}$ for all three cases. For $H a=0$ we have six layers for case $\mathrm{C}$, five layers for case $\mathrm{B}$, and three layers for case A. Over the range of $10 \leq H a \leq 17$, the decrease in the number of layers is the same for cases $B$ and C. The critical Hartmann numbers, $H a_{\mathrm{cr}}=20,26$, and 34 correspondings to a single layer for cases $\mathrm{A}$, $\mathrm{B}$, and $\mathrm{C}$ respectively. Consequently, the axial magnetic field affects a decrease in the number of resulting layers.

The diagram in the $\left(H a_{\mathrm{cr}}-\mathrm{Ri}\right)$ plane for all three cases when $\operatorname{Pr}=0.032$ presented in Fig. 16a gives the evolution of critical Hartmann number $H a_{\text {cr }}$ versus $R i$, for which the stratification fluid layers are suppressed and replaced by one fluid layer.
There are three separate curves, represent the limits of two-zone, i.e., the domains with and without stratification fluid layers. The blue curve with star symbols in Fig. 17a represents the boundary for case A. The red curve corresponding to case B. For case $\mathrm{C}$, the threshold of transition is plotted by the black curve with square symbols. In all cases, we have seen that the increase of the $R i$ causes the

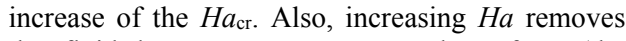
the fluid layers at a constant value of $R i$ (the layering disappears after the amplitude of $\mathrm{Ha}$ goes beyond a critical value). The critical values $\left(H a_{\text {cr }}\right)$ for case $\mathrm{C}$ are greater than those obtained in cases $\mathrm{A}$ and $\mathrm{B}$ for a fixed Richardson number.

Diagram, as shown in Fig. 17b, gives the evolution of transition from the multiple layers zone to the one layer zone for case $\mathrm{C}$ where Prandtl numbers are given by $\operatorname{Pr}=0.015,0.025$, and 0.032 , respectively. The zone above the black curve with square symbols consisting of one layer appears at $\operatorname{Pr}=0.032$ for the values of the critical Hartmann number $H a_{\mathrm{cr}}=19,26$, and 30.5 correspondings respectively to the values of the $\mathrm{Ri}=0.5,1.0$, and 2.0. We have seen that the critical Hartmann number evolves with increasing $R i$. On the other hand, $H a_{\mathrm{cr}}$ rises with increasing $P r$ for a fixed value of $R i$. Therefore, the values of $H a_{\mathrm{cr}}$ for case $P r=0.032$ are greater than those obtained in the cases of $P r=0.015$ and 0.025 . It is observed that the divergence between the two curves $(P r=0.032$ and $P r=0.025)$ increases with increasing $R i$. By contrast, it can be seen from Fig. 17b that the divergence between curves $P r=0.015$ and $P r=0.025$ is constant.

\section{CONCLUSION}

The effects of both buoyancy force and magnetic field on laminar vortex breakdown and fluid layers development through vertical annuli filled with a 

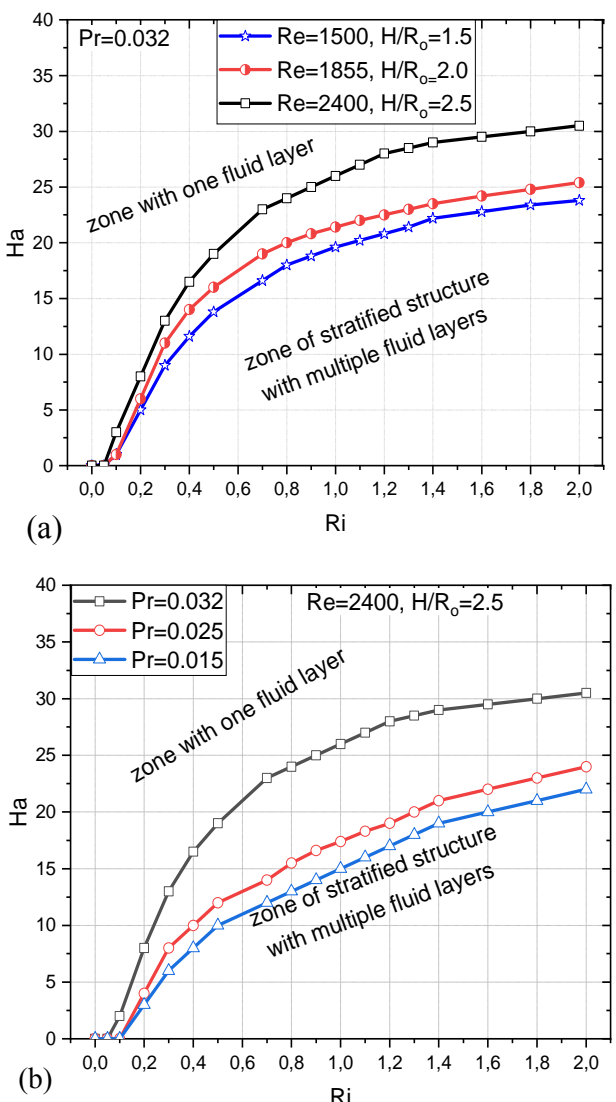

Fig. 17 (a-b) Curves of $\mathrm{Ha}_{\mathrm{cr}}$ versus Ri show the limits of the transition from the multiple fluid layers to the single-layer.

conducting viscous fluid with a bottom rotating disk have been numerically analyzed. The finite volume method has been used to capture the different vortex breakdowns in the hydrodynamic case and the stratified layers under the temperature stratification condition. Three configurations of Reynolds numbers and aspect ratios are compared, and three Prandtl numbers are analyzed. The main results obtained are as follows:

- It is found that when the annular gap is $R=0.9$, stability limits are moved to the left compared to the stability limits of $R=1.0$ obtained by Escudier (1984). It is also found that only a single vortex breakdown exists.

- In all cases $\mathrm{A}, \mathrm{B}$, and $\mathrm{C}$ investigated above, increasing Richardson number to 0.1 resulted in the suppression of vortex breakdown in which one layer occupied the annular gap.

- The competition between buoyancy and viscous forces occurs with the emergence of a clockwise flow near the hot top disk, and at a sufficiently high Richardson number, the flow tends to develop a fluid layered structure.

- The stratification layers increase with increasing $R i$ for all cases $\mathrm{A}, \mathrm{B}$, and $\mathrm{C}$.
- The average Nusselt number demonstrates a monotonically decreasing trend with increasing $R i$ in the onset of the layering, so the fluid layers act to insulate the hot annular gap. However, for the range of $R i$ considered the average Nusselt number increases with increasing Prandtl number.

- The Prandtl number and combination of (Reynolds number/aspect ratio) play an important role in the formation of fluid layers.

- With a magnetic field, the results showed that increasing $\mathrm{Ha}$ leads to suppress the vortex breakdown and to a decrease in the number of fluid layers

- Finally, the transition from multiple fluid layers to one fluid layer ( $\mathrm{Ha}_{\mathrm{cr}}$ ) evolves with increasing $R i$.

\section{ACKNOWLEDGEMENTS}

The Authors gratefully acknowledge the support of the General Directorate of Scientific Research and Technological Development (DGRSDT - Algeria).

\section{REFERENCES}

Bendjaghlouli, A., D. E. Ameziani, B. Mahfoud and L. Bouragbi (2019a). Magnetohydrodynamic counter rotating flow and heat transfer in a truncated conical container. Journal Thermophysics and Heat Transfer 33(3), 865874.

Bendjaghlouli, A., B. Mahfoud and D. E. Ameziani (2019b). Magnetohydrodynamic flow in a truncated conical enclosure. Journal of Thermal Engineering 14, 77-83.

Benjamin, T. B. (1962). Theory of Vortex Breakdown Phenomenon. Journal of Fluid Mechanics 14, 593-629.

Dash, S. C. and N. Singh. (Sept 2019). Influence of Axial Magnetic Field on Swirling Flow and Vortex Breakdown Zones in a Cylindrical Cavity with a Rotating Lid. International Journal of Applied Mechanics 11(6), 500-546.

Escudier, M. (1984). Observations of the flow produced in a cylindrical container by a rotating end wall. Experiments in Fluids 2, 189-196.

Herrada, M. A. and V. Shtern (2003). Control of vortex breakdown by temperature gradients. Physics of Fluids 2, 3468-3477.

Ismadi, M.P., P. Meunier. A. Fouras, A. And and K. Hourigan (2011). Experimental control of vortex breakdown by density effects. Physics of Fluids 23(3), 1-9.

Kakarantzas, S. C., L. Th. Benos. I. E. Sarris. B. Knaepen. A. P. Grecos and N. S. Vlachos (2017). MHD liquid metal flow and heat transfer between vertical coaxial cylinders under horizontal magnetic field. The International Journal of Heat and Fluid Flow $65,342-351$. 
H. Benhacine et al. / JAFM, Vol. 15, No. 2, pp. 563-577, 2022.

Kharicha, A., A. Alemany and D. Bornas (2005). Hydrodynamic study of a rotating MHD flow in a cylindrical cavity by ultrasound shift method. International Journal of Engineering Science 43(7), 589-615.

Laouari, A., B. Mahfoud. R. Bessaïh and A. Hadjadj (2021). Hydrodynamic instabilities in swirling flow under axial magnetic field. European Journal of Mechanics-B/Fluids 85, 245-260.

Mahfoud, B. (2021a). Effects of an Axial Magnetic Field on Vortex Breakdown and Fluid Layer. Journal of Applied Fluid Mechanics 14(6), 1741-1753.

Mahfoud, B. (2021b). Magnetohydrodynamic effect on vortex breakdown zones in coaxial cylinders. European Journal of MechanicsB/Fluids (89), 445-457.

Mahfoud, B., A. Bendjagloli and R. Bessaïh (2016). Magneto-hydrodynamic co-rotating flow in a vertical cylindrical container. Numerical Heat Transfer 69(9), 1051-1063.

Mahfoud, B., H. Benhacine. A. Laouari and A. Bendjaghlouli (2020). Magnetohydrodynamic effect on flow structures between coaxial cylinders heated from below. Journal of Thermophysics and Heat Transfer 34, 265274.

Mahfoud, B. and R. Bessaïh (2016). Magnetohydrodynamic counter-rotating flow in a cylindrical cavity. International Journal of Heat and mass Transfer 93, 175-185

Mahfoud, B. and R. Bessaïh (2012a). Stability of swirling flows with heat transfer in a cylindrical enclosure with co/counter-rotating end disks under an axial magnetic field. Numerical Heat Transfer, Part A 61(6), 463482.
Mahfoud, B. and R. Bessaïh (2012b). Oscillatory swirling flows in a cylindrical enclosure with co-/counter-rotating end disks submitted to a vertical temperature gradient. Fluid Dynamics \& Materials Processing 8, 1-26

Mahfoud, B., A. Laouari A. Hadjadj and H. Benhacine (2019). Counter-rotating flow in coaxial cylinders under an axial magnetic field. European Journal of Mechanics-B/Fluids 78, 139-146

Omi, Y. and R. Iwatsu (2005). Numerical study of swirling flows in cylindrical container with Co-/Counter-Rotating end disks under stable temperature difference. International Journal of Heat and Mass Transfer 48(23), 4854-4866.

Patankar, S. (1980). Numerical Heat Transfer and Fluid Flow. McGraw-Hill, New-York.

Quaresma, N. N., C. Cruz. N. da Cagney. R. M. Cotta and S. Balabani (2020). Effect of mixed convection on laminar vortex breakdown in a cylindrical enclosure with a rotating bottom plate. International Journal of Thermal Sciences $155,106399$.

Turan, O., S. Yigit and N. Chakraborty (2018). Effects of wall heating on laminar mixed convection in a cylindrical enclosure with a rotating end wall. International Journal of Thermal Sciences 131, 80-93.

Vogel, H. U. (1968). Experimentelle ergebnisse ueber die laminare stroemung in einem zylindrischen gehaeuse mit darin rotierender Sceibe. MIP Bericht, No. 6.

Yu, Y., B. W. Li and A. Thess (2013). The effect of a uniform magnetic field on vortex breakdown in a cylinder with rotating upper lid, Computers \& Fluids 88, 510-523. 